\title{
Resistance to Stem Rust Race TTKSK Maps to the rpg4/Rpg5 Complex of Chromosome $5 \mathrm{H}$ of Barley
}

\author{
B. J. Steffenson, Y. Jin, R. S. Brueggeman, A. Kleinhofs, and Y. Sun
}

First author: Department of Plant Pathology, University of Minnesota, St. Paul 55108; second author: United States Department of Agriculture-Agricultural Research Service, Cereal Disease Laboratory, St. Paul, MN 55108; third and fourth authors: Department of Crop and Soil Science, Washington State University, Pullman 99164; and fifth author: Monsanto Company, 8350 Minnegan Road, Waterman, IL 60556.

Accepted for publication 5 June 2009.

\begin{abstract}
Steffenson, B. J., Jin, Y., Brueggeman, R. S., Kleinhofs, A., and Sun, Y. 2009. Resistance to stem rust race TTKSK maps to the rpg4/Rpg5 complex of chromosome 5H of barley. Phytopathology 99:1135-1141.

Race TTKSK (Ug99) of the wheat stem rust pathogen (Puccinia graminis f. sp. tritici) is a serious threat to both wheat and barley production worldwide because of its wide virulence on many cultivars and rapid spread from eastern Africa. Line Q21861 is one of the most resistant barleys known to this race. To elucidate the genetics of resistance in this line, we evaluated the Q21861/SM89010 (Q/SM) doubled-haploid population for reaction to race TTKSK at the seedling stage. Segregation for resistance:susceptibility in Q/SM doubled-haploid lines fit a $1: 1$ ratio (58:71 with $\chi^{2}=1.31$ and $\left.P=0.25\right)$, indicating that a single gene in Q21861 confers resistance to race TTKSK. In previous studies, a recessive gene (rpg4) and a partially dominant gene (Rpg5)

were reported to control resistance to $P$. graminis f. sp. tritici race QCCJ and $P$. graminis f. sp. secalis isolate 92-MN-90, respectively, in Q21861. These resistance genes co-segregate with each other in the Q/SM population and were mapped to the long arm of chromosome $5 \mathrm{H}$. Resistance to race TTKSK also co-segregated with resistance to both rusts, indicating that the gene conferring resistance to race TTKSK also lies at the rpg4/Rpg5 locus. This result was confirmed through the molecular analysis of recombinants previously used to characterize loci conferring resistance to race QCCJ and isolate $92-\mathrm{MN}-90$. The $70-\mathrm{kb}$ region contains Rpg5 (a nucleotide-binding site leucine-rich repeat serine/threonineprotein kinase gene), rpg4 (an actin depolymerizing factor-like gene), and two other genes of unidentified function. Research is underway to resolve which of the genes are required for conferring resistance to race TTKSK. Regardless, the simple inheritance should make Q21861 a valuable source of TTKSK resistance in barley breeding programs.
\end{abstract}

Barley is an important crop in the Upper Midwest region of the United States and Prairie Provinces of Canada, with $>4.8$ million ha produced annually (13). Although stem rust (caused by Puccinia graminis f. sp. tritici Eriks. \& Henn. and P. graminis f. sp. secalis Eriks. \& Henn.) has historically been a problem on the crop in the past, it has not caused any widespread epidemics for over 70 years $(27,40)$. Since 1942 , effective control of stem rust has been achieved through the deployment of cultivars with the durable resistance gene Rpgl but other factors, such as a largely resistant wheat crop and barley's shorter maturation period coupled with the late arrival of rust inoculum to the northern planted crop, likely contributed to the long-lasting disease control (33). Rpgl is effective against many races of $P$. graminis $\mathrm{f}$. sp. tritici; however, some with virulence for this gene have been reported on cultivars from the field (33). One of the most recent cases involved QCCJ, a race thought to have originated in western Canada in the late 1980s (21,33). Race QCCJ became established in the Great Plains region in 1989 and damaged some barley fields planted to cultivars carrying Rpgl in both the United States and Canada (33). It has since become a minor component of the stem rust population in the United States and Canada after susceptible wheat cultivars were no longer grown in the central Great Plains. A new threat to barley is TTKSK (original described race designation: TTKS with isolate synonym of Ug99), a race first characterized from Uganda in 1999 (26). Race TTKSK has a wide virulence spectrum and is capable of attacking $>70 \%$ of the

Corresponding author: B. J. Steffenson; E-mail address: bsteffen@umn.edu

doi:10.1094/PHYTO-99-10-1135

(C) 2009 The American Phytopathological Society world's wheat cultivars (30). Recent data also indicate that it is virulent for many barley cultivars, including those bred with Rpg 1 $(34,36)$. Race TTKSK represents a serious threat to wheat and barley production worldwide because of its wide virulence, ability to cause severe yield loss (8), and rapid spread from Uganda. Since its initial discovery in 1999 (26), TTKSK has spread to other countries in eastern Africa (Kenya, Ethiopia, and Sudan), crossed the Red Sea into Yemen in 2006, and is now confirmed in Iran $(23,30,42)$. It may only be a short time before it spreads into the vast cereal production areas of southern Asia and beyond. In the short term, fungicides offer the best control solution where no resistant cultivars are available; however, this strategy is prohibitively expensive for many of the region's subsistence growers and raises some environmental concerns. The deployment of resistant cereal crops is the most effective strategy for combating the threat posed by race TTKSK. A global effort to breed wheat for resistance to race TTKSK was launched after an international expert panel convened to address the problem in 2005. Through this initiative, now called the Borlaug Global Rust Initiative, significant progress has been made in identifying new and native sources of stem rust resistance and transferring the resistance genes into adapted wheat cultivars $(1,30,31)$. Comparatively little research has been advanced on barley, due to its smaller worldwide acreage and lower rank as a food crop for people. Yet, in the very countries where TTKSK now exists (Ethiopia and Yemen), barley is one of the most important food crops, especially for people living at high elevation (12). Wheat and barley are often cultivated in the same areas. To effectively control stem rust, it is essential that both crops be bred for resistance, otherwise inoculum from one crop could initiate epidemics on the other crop. Furthermore, the effective population size of $P$. graminis $\mathrm{f}$. sp. 
tritici on both crops must be held in check, otherwise the chance for dangerous mutants to arise will increase (28).

In barley, at least eight genes for resistance to stem rust have been described. Those identified on the basis of their reaction to races of the wheat stem rust pathogen, $P$. graminis f. sp. tritici, include Rpgl derived from cvs. Peatland (CIho 5267), Chevron (CIho 1111), and Kindred (CIho 6969) (25,29,33); Rpg2 from Hietpas 5 (CIho 7124) (24); Rpg3 from PI 382313 (14,15); rpg4 from Q21861 (PI 584766) (17); and Rpg6 from 212Y1, a barley line with a Hordeum bulbosum introgression (9). Another gene, aside from Rpgl, was identified in Peatland (designated as RpgU) and confers moderate resistance to race QCCJ in the field (10). Three genes have been identified on the basis of their reaction to the rye stem rust pathogen, $P$. graminis f. sp. secalis. A single dominant gene for resistance was identified in the barley accession Skinless (20), and a single recessive gene ( $r p g B H$, formerly designated $S$ gene) was found in Black Hulless (CIho 666) (37). Additionally, a partially dominant gene for resistance to $P$. graminis f. sp. secalis was reported in Q21861 by Sun et al. (39) and later provisionally designated as $\operatorname{Rpg} Q$ (38). This gene was thought to be the same as rpg4 because the resistance conferred by $\operatorname{Rpg} Q$ to rye stem rust co-segregated with resistance to wheat stem rust race QCCJ conferred by rpg4 (39). However, recent high-resolution mapping efforts have shown that $\operatorname{Rpg} Q$ is a different but closely linked gene to rpg4 on the long arm of chromosome $5 \mathrm{H}$ and was given the new locus symbol of Rpg5 (3).

In barley, research is urgently needed on identifying sources of resistance to race TTKSK and transferring resistance genes into adapted breeding lines. Preliminary evaluations of Hordeum germplasm have identified several sources of resistance to race TTKSK in various cultivars, landraces, and wild barley accessions $(34,36)$. Breeding line Q21861 was found to be one of the most resistant barley lines to race TTKSK at both the seedling stage in greenhouse and at the adult plant stage in the field (B. Steffenson and Y. Jin, unpublished). Information on the genetics of TTKSK resistance in Q21861 is important for developing the most efficient strategy for stem rust resistance breeding. Thus, the objective of this study was to determine the number and chromosomal location of genes controlling resistance to race TTKSK in Q21861.

\section{MATERIALS AND METHODS}

Plant materials. The doubled-haploid $(\mathrm{DH})$ population of Q21861/SM89010 (Q/SM) was used for this study. It was produced by anther culture from $\mathrm{F}_{1}$ plants of the two parents and consists of 129 lines (35). Q21861 is a two-rowed breeding line of unknown parentage that was originally selected in 1983 from a breeding nursery established at Centro Internacional de Mejoramiento de Maíz y Trigo in Mexico. It carries the stem rust resistance genes Rpg1, rpg4, and Rpg5 $(3,17,35)$ as well as other disease resistance genes (11,35). SM89010 is a two-rowed malting barley line from the University of Saskatchewan breeding program. It was derived from the cross Nairn $\times$ Manley and is susceptible to stem rust.

Plant growth conditions, inoculation protocol, and infection/incubation period. Evaluations of the Q/SM population for resistance to race TTKSK were done at the United States Department of Agriculture-Agricultural Research Service Cereal Disease Laboratory during the winter months. Three to five seeds from each $\mathrm{DH}$ line and the parents were planted in plastic cones $(21 \mathrm{~cm}$ in depth and $3.8 \mathrm{~cm}$ in diameter) filled with a 50:50 mixture of soil and Metro Mix 200 (vermiculite, peat moss, perlite, and sand) and fertilized with Osmocote 14-14-14 (Scott's Company, Marysville, OH) (1.4 g/cone) and Peters Dark Weather 15-0-15 (Scott's Company) (150 g per 3.78 liter at 1/16 dilution). Plants were grown in a greenhouse at $22^{\circ} \mathrm{C}$ with supplemental lighting provided by $1,000-\mathrm{W}$ sodium vapor lamps for $14 \mathrm{~h}$ per day. Isolate 04KEN156/04 of race TTKSK was initially increased on a susceptible wheat host, collected, desiccated, and stored in tubes at $-80^{\circ} \mathrm{C}$ until needed. On the day of inoculation, the tubes of rust were heat shocked at $45^{\circ} \mathrm{C}$ for $10 \mathrm{~min}$, rehydrated in $80 \%$ $\mathrm{KOH}$, placed in gelatin capsules containing a lightweight mineral oil carrier (Soltrol 170; Phillips Petroleum, Bartlesville, OK), and applied to 9-day-old plants with fully expanded primary leaves. The concentration of inoculum used was $4.28 \mathrm{mg} / \mathrm{ml}$ of lightweight mineral oil. Inoculation protocols were as described by Sun and Steffenson (38), except that $0.004 \mathrm{mg}$ of rust was applied per plant instead of $0.035 \mathrm{mg}$. After inoculation, plants were placed in mist chambers at $21^{\circ} \mathrm{C}$ for $16 \mathrm{~h}$ in the dark. During this time, the plants were kept moist by periodic misting from ultrasonic humidifiers. Then, light was provided by fluorescent bulbs, and the chamber doors were partially opened to reduce the build up of heat. After 3 to $4 \mathrm{~h}$, the leaf surfaces were completely dry, and plants were returned to the greenhouse under the conditions described above.

The experiment was planted in a completely randomized design with one replicate and was repeated once. Three to five plants were assessed per replicate. A few DH lines gave variable reactions between experiments and were tested again in two additional experiments.

Disease assessment. Fourteen to seventeen days after inoculation, the infection types (ITs) on each DH line and the parents were assessed using a 0 to 4 scale. The IT scale used for barley is a modification of the one developed for wheat by Stakman et al. (32) and is based primarily on uredinial size as described by Miller and Lambert (22). Barley frequently exhibits two or more ITs on a single leaf (i.e., a mesothetic reaction) when infected with $P$. graminis (38); thus, all of the ITs observed on each DH line were recorded in order of their prevalence. To simplify the presentation of data, the two most common ITs (i.e., the IT mode) are presented in Table 1. Comparisons were made on the basis of the IT mode because the two most common ITs usually comprised $>85 \%$ of all those observed on individual DH lines. Additionally, the lowest and highest ITs (i.e., the IT range) observed also are given to indicate the degree of variation in stem rust reactions. Classification of progeny into resistant and susceptible categories was based on the reaction of the parents to race TTKSK. The genetics of resistance to both $P$. graminis f. sp. tritici (races QCCJ and MCCF) and $P$. graminis f. sp. secalis (isolate 92-MN-90) was previously investigated in the Q/SM population $(35,39)$. Raw, unpublished IT data from these studies are presented in Table 1. These IT data were compared with those collected in this study, by inspection, to race TTKSK to assess the agreement of general reaction phenotypes (i.e., resistance versus susceptibility).

Genetic and physical mapping. Restriction fragment length polymorphism (RFLP) markers were developed for the Q/SM population as described by Druka et al. (6). Sequence tag site (STS) markers were developed using the Q21861 genomic sequence from the rpg4/Rpg5 region (accession no. EU878778) (3). Regions with polymorphism between Q21861 and SM89010 were utilized for polymerase chain reaction (PCR)-based marker development. The simple sequence repeat (SSR) markers were developed from cv. Morex bacterial artificial chromosome (BAC) sequence (accession no. EU812563). Primer sequences for STS and SSR markers are shown in Table 2. A single nucleotide polymorphism (SNP) was scored in the RB SSR1 amplicon by sequencing the PCR product from the $\mathrm{Q} / \mathrm{SM}$ recombinants. The RB SSR1 amplicon length is identical between Q21861 and SM89010; however, SM89010 contains an additional CAA repeat and is missing one CAT repeat compared with Q21861. Molecular analysis of the rpg4/Rpg5 region used seven recombinants from $\mathrm{Q} / \mathrm{SM}$ based on 129 gametes within the flanking region defined by RFLP markers ABG391 and MWG740. BAC physical maps were developed according to Druka et al. (6) and Brueggeman et al. (3). 


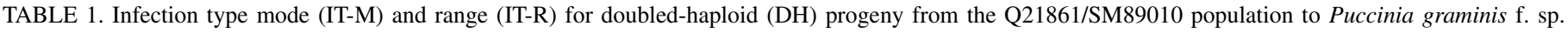
tritici races TTKSK, QCCJ, and MCCF and P. graminis f. sp. secalis isolate 92-MN-90 at the seedling stage

\begin{tabular}{|c|c|c|c|c|c|c|c|c|c|c|c|c|}
\hline \multirow[b]{2}{*}{ DH line } & \multicolumn{3}{|c|}{ TTKSK } & \multicolumn{3}{|c|}{ 92-MN-90 } & \multicolumn{3}{|c|}{ QCCJ } & \multicolumn{3}{|c|}{$\mathrm{MCCF}$} \\
\hline & IT-M & IT-R & Allele & IT-M & IT-R & Allele & IT-M & IT-R & Allele & IT-M & IT-R & Allele \\
\hline Q21861 & 0 & 0 & rpg4/Rpg5 & 00 & 0 to 0 & Rpg5 & 00 & 0 to 1 & rpg4 & $0 ; 1-$ & 0 ; to 1 & Rpg1 \\
\hline SM89010 & 32 & 1 to 3 & Rpg4/rpg5 & $23-$ & 2 to $3-$ & rpg 5 & $33-$ & 2 to $3+$ & Rpg4 & $3-3$ & $3-$ to 3 & rpgl \\
\hline QSM001 & $33+$ & 3 to $3+$ & Rpg4/rpg5 & $3-32$ & 1 to 3 & rpg 5 & $33-$ & 2 to $3+$ & Rpg4 & 10 & $0 ;$ to 2 & Rpg1 \\
\hline QSM002 & $0 ; 1$ & $0 ;$ to 1 & rpg4/Rpg5 & 00 & 0 to 0 & Rpg5 & 00 & 0 to 1 & rpg4 & $3-3$ & $3-$ to 3 & rpgl \\
\hline QSM003 & 0 & 0 & rpg4/Rpg5 & $0 ; 1$ & 0 to 1 & Rpg5 & $0 ; 1$ & 0 to 1 & rpg4 & $33-$ & $3-$ to 3 & rpgl \\
\hline QSM004 & 3 & 3 & Rpg4/rpg5 & $23-$ & 2 to $3-$ & rpg5 & $33-$ & 2 to $3+$ & Rpg4 & $33-$ & $3-$ to $3+$ & rpgl \\
\hline QSM005 & $0 ; 1-$ & 0 ; to $1-$ & rpg4/Rpg5 & 00 & 0 to 0 & Rpg5 & 00 & 0 to 1 & rpg4 & $0 ; 1-$ & 0 to $1-$ & Rpg1 \\
\hline QSM008 & $0 ; 1-$ & 0 ; to $1-$ & rpg4/Rpg5 & 00 & 0 to 0 & Rpg 5 & 00 & 0 to 1 & rpg4 & $0 ; 1-$ & 0 ; to $1-$ & Rpg1 \\
\hline QSM010 & $3-2$ & 2 to $3-$ & Rpg4/rpg5 & 213- & 1 to $3-$ & rpg5 & $3-3$ & 2 to 3 & Rpg4 & $0 ; 1$ & $0 ;$ to 1 & Rpg1 \\
\hline QSM011 & 32 & 2 to $3+$ & Rpg4/rpg5 & 213- & 1 to $3-$ & rpg 5 & $33-$ & 2 to $3+$ & Rpg4 & $33-$ & $3-$ to 3 & rpg1 \\
\hline QSM012 & 3 & $3-$ to 3 & Rpg4/rpg5 & $3-2$ & 2 to $3-$ & rpg 5 & $33-$ & 2 to $3+$ & Rpg4 & $12-$ & 1 to $2-$ & Rpg1 \\
\hline QSM013 & $3+$ & $3+$ & Rpg4/rpg5 & $3-2$ & 1 to $3-$ & rpg 5 & $33+$ & 3 to $3+$ & Rpg4 & 21 & 1 to $3-$ & Rpg1 \\
\hline QSM014 & $0 ; 1$ & $0 ;$ to 1 & rpg4/Rpg5 & 00 & 0 to 0 & Rpg5 & $0 ; 1$ & 0 to 2 & rpg4 & $3-3$ & $3-$ to 3 & rpgl \\
\hline QSM015 & $0 ; 1-$ & 0 ; to $1-$ & rpg4/Rpg5 & $0 ; 1$ & 0 ; to 1 & Rpg 5 & $0 ; 1$ & 0 to 2 & rpg4 & $33-$ & 2 to 3 & rpgl \\
\hline QSM016 & $0 ; 1-$ & 0 ; to $1-$ & rpg4/Rpg5 & $0 ; 1$ & 0 ; to 1 & Rpg 5 & 00 & 0 to 0 & rpg4 & $3-3$ & $3-$ to 3 & rpgl 1 \\
\hline QSM017 & $3-$ & $3-$ & Rpg4/rpg5 & 213- & 1 to 3 & rpg5 & $3-2$ & 2 to 3 & Rpg4 & $33-$ & $3-$ to $3+$ & rpgl \\
\hline QSM018 & $0 ; 1$ & 0 ; to 1 & rpg4/Rpg5 & $0 ; 1$ & 0 ; to 1 & Rpg5 & 0 & 0 to 1 & rpg4 & $3-3$ & $3-$ to 3 & rpgl 1 \\
\hline QSM019 & 3 & $3-$ to 3 & Rpg4/rpg5 & $3-2$ & 2 to $3-$ & rpg5 & $33-$ & $3-$ to $3+$ & Rpg4 & $33-$ & $3-$ to $3+$ & rpgl 1 \\
\hline QSM020 & $0 ; 1$ & 0 ; to 1 & rpg4/Rpg5 & 00 & 0 to 0 & Rpg5 & 00 & 0 to 1 & rpg4 & $33-$ & $3-$ to 3 & rpgl \\
\hline QSM023 & 3 & 3 & Rpg4/rpg5 & $3-2$ & 2 to 3 & rpg5 & $33+$ & $3-$ to $3+$ & Rpg4 & $33-$ & $3-$ to 3 & rpgl \\
\hline QSM024 & $0 ; 1$ & 0 ; to 1 & rpg4/Rpg5 & 00 & 0 to 0 & Rpg5 & 00 & 0 to 0 & rpg4 & 10 & $0 ;$ to 2 & Rpg1 \\
\hline QSM026 & 0 & 0 & rpg4/Rpg5 & 00 & 0 to 0 & Rpg 5 & 00 & 0 to 0 & rpg4 & $0 ; 1-$ & 0 ; to $1-$ & Rpg1 \\
\hline QSM028 & 3 & 3 & Rpg4/rpg5 & $3-2$ & 2 to $3-$ & rpg5 & $33+$ & $3-$ to $3+$ & Rpg4 & $0 ; 1-$ & 0 ; to $1-$ & Rpg1 \\
\hline QSM029 & 10 & $0 ;$ to 1 & rpg4/Rpg5 & $0 ; 1$ & $0 ;$ to 2 & Rpg5 & $0 ; 1$ & 0 to 2 & rpg4 & $33-$ & 2 to 3 & rpgl \\
\hline QSM030 & $3+$ & $3-$ to $3+$ & Rpg4/rpg5 & $23-1$ & 1 to 3 & rpg5 & $33-$ & 2 to $3+$ & Rpg4 & $0 ; 1$ & $0 ;$ to 1 & Rpg1 \\
\hline QSM031 & $33+$ & 3 to $3+$ & Rpg4/rpg5 & 213- & 0 ; to 3- & rpg5 & $33+$ & 2 to $3+$ & Rpg4 & $33-$ & $3-$ to 3 & rpgl \\
\hline QSM033 & $0 ; 1$ & 0 ; to 1 & rpg4/Rpg5 & 00 & 0 to $1-$ & Rpg 5 & $0 ; 1$ & 0 to 2 & rpg4 & $3-3$ & $3-$ to 3 & rpgl 1 \\
\hline QSM034 & 3 & 3 & Rpg4/rpg5 & $23-1$ & 1 to $3-$ & rpg5 & $33+$ & $3-$ to $3+$ & Rpg4 & $33-$ & $3-$ to $3+$ & rpgl \\
\hline QSM035 & 3 & 3 & Rpg4/rpg5 & 213- & 1 to $3-$ & rpg5 & $3-3$ & 2 to $3+$ & Rpg4 & $3-3$ & $3-$ to 3 & rpgl \\
\hline QSM036 & 3 & 3 & Rpg4/rpg5 & $23-1$ & 1 to $3-$ & rpg5 & $33-$ & 2 to $3+$ & Rpg4 & $33-$ & $3-$ to 3 & rpgl 1 \\
\hline QSM037 & $0 ; 1-$ & 0 ; to $1-$ & rpg4/Rpg5 & $0 ; 1$ & 0 to 1 & Rpg5 & $0 ; 1$ & $0 ;$ to 2 & rpg4 & $33-$ & $3-$ to 3 & rpgl \\
\hline QSM038 & 3 & 3 & Rpg4/rpg5 & $3-21$ & 1 to $3-$ & rpg5 & $33-$ & $3-$ to $3+$ & Rpg4 & $33-$ & $3-$ to 3 & rpgl \\
\hline QSM039 & $0 ; 1-$ & 0 ; to $1-$ & rpg4/Rpg5 & $0 ; 1$ & 0 ; to 1 & Rpg5 & 00 & 0 to $1-$ & rpg4 & $0 ; 1-$ & 0 ; to $1-$ & Rpg1 \\
\hline QSM041 & $0 ; 1-$ & $0 ;$ to 1 & rpg4/Rpg5 & 00 & 0 to 0 & Rpg5 & 00 & 0 to 0 & rpg4 & 10 & 0 ; to $2-$ & Rpg1 \\
\hline QSM042 & $0 ; 1$ & 0 ; to 1 & rpg4/Rpg5 & 00 & 0 to 0 & Rpg 5 & 00 & 0 to 1 & rpg4 & $3-3$ & 2 to 3 & rpgl \\
\hline QSM043 & $33-$ & $3-$ to 3 & Rpg4/rpg5 & $23-1$ & 1 to $3-$ & rpg5 & $33-$ & 2 to $3+$ & Rpg4 & $33-$ & $3-$ to $3+$ & rpgl \\
\hline QSM045 & 3 & $3-$ to $3+$ & Rpg4/rpg5 & $23-1$ & 1 to $3-$ & rpg5 & $33+$ & $3-$ to $3+$ & Rpg4 & 12 & 1 to 2 & Rpg1 \\
\hline QSM046 & $0 ; 1$ & $0 ;$ to 1 & rpg4/Rpg5 & 00 & 0 to 0 & Rpg5 & $0 ; 1$ & $0 ;$ to 2 & rpg4 & $0 ; 1$ & 0 ; to $2-$ & Rpg1 \\
\hline QSM047 & $33+$ & 3 to $3+$ & Rpg4/rpg5 & $3-2$ & 2 to $3-$ & rpg5 & $33+$ & 2 to $3+$ & Rpg4 & $23-$ & 1 to $3-$ & Rpg1 \\
\hline QSM048 & $33+$ & 3 to $3+$ & Rpg4/rpg5 & 21 & 1 to 2 & rpg 5 & $33+$ & 2 to $3+$ & Rpg4 & $33-$ & $3-$ to 3 & rpgl \\
\hline QSM049 & $0 ; 1-$ & 0 ; to $1-$ & rpg4/Rpg5 & 00 & 0 to 0 & Rpg5 & $0 ; 1$ & 0 to 1 & rpg4 & $3-3$ & $3-$ to 3 & rpgl 1 \\
\hline QSM050 & $0 ; 1$ & $0 ;$ to 1 & rpg4/Rpg5 & 00 & 0 to 0 & Rpg 5 & 00 & 0 to $0 ; 1$ & rpg4 & $33-$ & $3-$ to 3 & rpgl \\
\hline QSM052 & $0 ; 1-$ & 0 ; to $1-$ & rpg4/Rpg5 & 00 & 0 to $1-$ & Rpg5 & 00 & 0 to 0 & rpg4 & $3-3$ & 2 to 3 & rpgl \\
\hline QSM054 & 0 & 0 & rpg4/Rpg5 & 00 & 0 to 0 & Rpg5 & $0 ; 1$ & $0 ;$ to 2 & rpg4 & $0 ; 1$ & 0 ; to $2-$ & Rpg1 \\
\hline QSM055 & $33-$ & $3-$ to 3 & Rpg4/rpg5 & 213- & 1 to $3-$ & rpg5 & $33-$ & 2 to $3+$ & Rpg4 & $0 ; 1$ & $0 ;$ to 1 & Rpg1 \\
\hline QSM056 & $0 ; 1$ & 0 ; to 1 & rpg4/Rpg5 & 00 & 0 to 0 & Rpg5 & $0 ; 1$ & $0 ;$ to 2 & rpg4 & $33-$ & $3-$ to 3 & rpgl \\
\hline QSM057 & 10 & 0 ; to 1 & rpg4/Rpg5 & 00 & 0 to 0 & Rpg5 & $0 ; 1$ & $0 ;$ to 2 & rpg4 & $3-3$ & $3-$ to 3 & rpgl \\
\hline QSM058 & $33+$ & 3 to $3+$ & Rpg4/rpg5 & $3-3$ & $3-$ to 3 & rpg5 & $33-$ & 2 to $3+$ & Rpg4 & 21 & 1 to $3-$ & Rpg1 \\
\hline QSM059 & $33-$ & $3-$ to 3 & Rpg4/rpg5 & $23-1$ & 1 to 3 & rpg5 & $33+$ & 2 to $3+$ & Rpg4 & $33-$ & $3-$ to 3 & rpgl \\
\hline QSM060 & $3-$ & $3-$ & Rpg4/rpg5 & $23-1$ & 1 to $3-$ & rpg 5 & $33-$ & 2 to $3+$ & Rpg4 & $33-$ & $3-$ to 3 & rpgl 1 \\
\hline QSM061 & 3 & $3-$ to $3+$ & Rpg4/rpg5 & $3-23$ & 1 to 3 & rpg5 & $33+$ & 2 to $3+$ & Rpg4 & $33-$ & 2 to 3 & rpgl \\
\hline QSM062 & 3 & $3-$ to $3+$ & Rpg4/rpg5 & 213- & 1 to $3-$ & rpg 5 & $33+$ & $3-$ to $3+$ & Rpg4 & 12 & 1 to $3-$ & Rpg1 \\
\hline QSM063 & 3 & $2+$ to 3 & Rpg4/rpg5 & $23-1$ & 1 to $3-$ & rpg 5 & $33-$ & $3-$ to $3+$ & Rpg4 & $0 ; 1$ & $0 ;$ to 1 & Rpg1 \\
\hline QSM065 & $3+$ & $3+$ & Rpg4/rpg5 & $3-2$ & 2 to $3-$ & rpg5 & $3+3$ & 3 to $3+$ & Rpg4 & $0 ; 1$ & $0 ;$ to 2 & Rpg1 \\
\hline QSM066 & $0 ; 1$ & 0 ; to 1 & rpg4/Rpg5 & 00 & 0 to 0 & Rpg5 & $0 ; 1$ & $0 ;$ to 1 & rpg4 & $0 ; 1$ & 0 ; to 1 & Rpg1 \\
\hline QSM067 & 10 & 0 ; to 2 & rpg4/Rpg5 & 00 & 0 to 0 & Rpg5 & $0 ; 1$ & $0 ;$ to 2 & rpg4 & $33-$ & $3-$ to 3 & rpgl \\
\hline QSM068 & 3 & 3 & Rpg4/rpg5 & $23-1$ & 1 to $3-$ & rpg5 & $33-$ & 2 to $3+$ & Rpg4 & $33-$ & $3-$ to 3 & rpgl 1 \\
\hline QSM069 & 3 & 3 & Rpg4/rpg5 & $3-3$ & 2 to 3 & rpg 5 & $33+$ & $3-$ to $3+$ & Rpg4 & 10 & 0 ; to $2-$ & Rpg1 \\
\hline QSM070 & 0 & 0 & rpg4/Rpg5 & 00 & 0 to 0 & Rpg 5 & $0 ; 1$ & $0 ;$ to 2 & rpg4 & $33-$ & $3-$ to 3 & rpgl \\
\hline QSM071 & $3-$ & $3-$ & Rpg4/rpg5 & $23-1$ & 1 to $3-$ & rpg5 & $33-$ & 2 to 3 & Rpg4 & $0 ; 1$ & $0 ;$ to 1 & Rpg1 \\
\hline QSM073 & 3 & 3 & Rpg4/rpg5 & $3-2$ & 2 to $3-$ & rpg5 & $33+$ & 3 to $3+$ & Rpg4 & 21 & 1 to $3-$ & Rpg1 \\
\hline QSM074 & 3 & 3 to $3+$ & Rpg4/rpg5 & $3-21$ & 1 to $3-$ & rpg5 & $33-$ & 2 to $3+$ & Rpg4 & 21 & 1 to 2 & Rpg1 \\
\hline QSM075 & $0 ; 1$ & 0 ; to $2-$ & rpg4/Rpg5 & $0 ; 1-$ & 0 to $1-$ & Rpg5 & 21 & 0 ; to $3-$ & rpg4 & $33-$ & $3-$ to 3 & rpgl \\
\hline QSM076 & 3 & 3 & Rpg4/rpg5 & $3-21$ & 0 ; to 3- & $\operatorname{rpg} 5$ & $33-$ & 2 to $3+$ & Rpg4 & $3-2$ & 2 to 3 & Rpg1 \\
\hline QSM077 & 3 & 3 & Rpg4/rpg5 & 213- & 1 to $3-$ & $\operatorname{rpg} 5$ & $33+$ & 2 to $3+$ & Rpg4 & $33-$ & $3-$ to 3 & rpgl \\
\hline QSM078 & $33-$ & $3-$ to 3 & Rpg4/rpg5 & $23-1$ & 1 to $3-$ & rpg5 & $3-3$ & 2 to 3 & Rpg4 & $3-3$ & $3-$ to $3+$ & rpgl \\
\hline
\end{tabular}

a ITs are based on the 0 to 4 scale of Stakman et al. (32) as modified for barley by Miller and Lambert (22). The two (or three in the case of 92-MN-90) most common ITs observed on accessions (i.e., IT-M) are given in addition to the lowest and highest types observed (i.e., IT-R). Allele = recognized allele. The raw IT data for P. graminis f. sp. secalis isolate $92-\mathrm{MN}-90$ and the two $P$. graminis f. sp. tritici races (QCCJ and MCCF) have not been previously published, but are from the studies of Sun et al. (39) and Steffenson et al. (35), respectively. Interactions highlighted in gray indicate the presence of the respective resistance alleles in the QSM line. 


\section{RESULTS AND DISCUSSION}

Q21861 was highly resistant to race TTKSK and consistently exhibited a very low IT mode of 0; (Table 1). In contrast, SM89010 was susceptible, exhibiting an IT mode of 32. Q/SM DH progeny could be easily classified into two distinct groups based on their IT mode. The resistant group included 58 progeny with IT modes from 0 ; to $0 ; 1$ (rarely 10; and 12), and the susceptible group included 71 progeny with IT modes from 3- to 3+ and combinations thereof (rarely 23- and 3-2). The 58:71 segregation for resistance:susceptibility in the $\mathrm{Q} / \mathrm{SM} \mathrm{DH}$ population fit a $1: 1$ ratio $\left(\chi^{2}=1.31, P=0.25\right)$,

TABLE 1. (continued from preceding page)

\begin{tabular}{|c|c|c|c|c|c|c|c|c|c|c|c|c|}
\hline \multirow[b]{2}{*}{ DH line } & \multicolumn{3}{|c|}{ TTKSK } & \multicolumn{3}{|c|}{ 92-MN-90 } & \multicolumn{3}{|c|}{ QCCJ } & \multicolumn{3}{|c|}{$\mathrm{MCCF}$} \\
\hline & IT-M & IT-R & Allele & IT-M & IT-R & Allele & IT-M & IT-R & Allele & IT-M & IT-R & Allele \\
\hline QSM079 & 3 & 2 to $3+$ & Rpg4/rpg5 & $3-23$ & 1 to $3-$ & $\operatorname{rpg} 5$ & $33+$ & 2 to $3+$ & Rpg4 & $33-$ & $3-$ to $3+$ & rpgl \\
\hline QSM080 & 0 & 0 & rpg4/Rpg5 & 00 & 0 to 0 & Rpg5 & 00 & 0 to 0 & rpg4 & $0 ; 1-$ & 0 ; to $1-$ & Rpg1 \\
\hline QSM082 & 3 & 3 & Rpg4/rpg5 & $213-$ & 1 to $3-$ & $\operatorname{rpg} 5$ & $33+$ & $3-$ to $3+$ & Rpg4 & $33-$ & $3-$ to $3+$ & rpgl \\
\hline QSM083 & 3 & $3-$ to $3+$ & Rpg4/rpg5 & $3-3$ & 2 to $3-$ & rpg 5 & $3-3$ & 2 to $3+$ & Rpg4 & 10 & 0 ; to $2-$ & Rpg1 \\
\hline QSM084 & $33-$ & $3-$ to 3 & Rpg4/rpg5 & 12 & $0 ;$ to 2 & rpg 5 & $3-2$ & 2 to $3-$ & Rpg4 & $0 ; 1-$ & $0 ;$ to 1 & Rpg1 \\
\hline QSM085 & $33-$ & $3-$ to 3 & Rpg4/rpg5 & $33-$ & 2 to 3 & rpg5 & $33+$ & 2 to $3+$ & Rpg4 & $0 ; 1-$ & 0 ; to 1 & Rpg1 \\
\hline QSM086 & 0 & 0 & rpg4/Rpg5 & 00 & 0 to 0 & Rpg 5 & 00 & 0 to 1 & rpg4 & $33-$ & $3-$ to 3 & rpgl \\
\hline QSM087 & $0 ; 1$ & 0 ; to 1 & rpg4/Rpg5 & 00 & 0 to 0 & Rpg 5 & $0 ; 1$ & 0 to 2 & rpg4 & $33-$ & $3-$ to 3 & rpgl \\
\hline QSM088 & 3 & $3-$ to 3 & Rpg4/rpg5 & $213-$ & 1 to $3-$ & $\operatorname{rpg} 5$ & $3-3$ & 2 to $3+$ & Rpg4 & $3-3$ & $3-$ to 3 & rpg1 \\
\hline QSM089 & $0 ; 1-$ & 0 ; to $1-$ & rpg4/Rpg5 & $0 ; 1-$ & 0 to $1-$ & Rpg5 & 00 & 0 to $2-$ & rpg4 & $12-$ & 0 ; to 2 & Rpg1 \\
\hline QSM090 & $23-$ & 2 to $3-$ & Rpg4/rpg5 & $23-1$ & 1 to $3-$ & $\operatorname{rpg} 5$ & $33+$ & $3-$ to $3+$ & Rpg4 & $3-3$ & $3-$ to 3 & rpgl \\
\hline QSM091 & 3 & 3 & Rpg4/rpg5 & $3-2$ & 2 to $3-$ & $\operatorname{rpg} 5$ & $33-$ & 2 to $3+$ & Rpg4 & 2 & 1 to 3 & Rpgl \\
\hline QSM093 & 0 & 0 & rpg4/Rpg 5 & 00 & 0 to 0 & Rpg 5 & 00 & 0 to 1 & rpg4 & $3-3$ & 2 to 3 & rpgl \\
\hline QSM094 & $33+$ & 3 to $3+$ & Rpg4/rpg5 & $3-21$ & 1 to $3-$ & $\operatorname{rpg} 5$ & $33-$ & 2 to $3+$ & Rpg4 & 10 & 0 ; to 1 & Rpg1 \\
\hline QSM095 & $0 ; 1-$ & 0 ; to $1-$ & rpg4/Rpg5 & 00 & 0 to 0 & Rpg5 & $0 ; 1$ & $0 ;$ to 1 & rpg4 & $0 ; 1-$ & $0 ;$ to 1 & Rpg1 \\
\hline QSM097 & $0 ; 1$ & 0 ; to 1 & rpg4/Rpg5 & 0 & 0 ; to $1-$ & Rpg 5 & 00 & 0 to $1-$ & rpg4 & $0 ; 1-$ & 0 ; to $1-$ & Rpg1 \\
\hline QSM098 & $0 ; 1$ & 0 ; to 1 & rpg4/Rpg5 & $0 ; 1$ & $0 ;$ to 1 & $\operatorname{Rpg} 5$ & 00 & 0 to 1 & rpg4 & $0 ; 1$ & 0 ; to $1-$ & Rpg1 \\
\hline QSM099 & $3-$ & $3-$ & Rpg4/rpg5 & $3-3$ & 2 to 3 & rpg5 & $33-$ & 2 to $3+$ & Rpg4 & $33-$ & $3-$ to 3 & rpgl \\
\hline QSM100 & $0 ; 1-$ & 0 ; to $1-$ & rpg4/Rpg5 & $0 ; 1$ & 0 ; to 1 & Rpg5 & 00 & 0 to 1 & rpg4 & $0 ; 1-$ & 0 ; to 1 & Rpg1 \\
\hline QSM101 & $0 ; 1$ & $0 ;$ to 1 & rpg4/Rpg5 & $0 ; 1$ & 0 ; to 1 & Rpg 5 & 00 & 0 to 1 & rpg4 & $0 ; 1-$ & $0 ;$ to 1 & Rpg1 \\
\hline QSM103 & $0 ; 1$ & 0 ; to 1 & rpg4/Rpg5 & $0 ; 1$ & 0 to 1 & Rpg 5 & $0 ; 1$ & $0 ;$ to 2 & rpg4 & 10 & 0 ; to $2-$ & Rpgl \\
\hline QSM104 & $3-$ & $3-$ & Rpg4/rpg5 & $3-2$ & 2 to $3-$ & rpg5 & $33-$ & 2 to $3+$ & Rpg4 & $33-$ & $3-$ to 3 & rpgl \\
\hline QSM105 & $33-$ & 2 to 3 & Rpg4/rpg5 & $213-$ & 1 to $3-$ & rpg 5 & $3-2$ & 2 to $3+$ & Rpg4 & $3-3$ & $3-$ to 3 & rpgl \\
\hline QSM106 & $0 ; 1$ & 0 ; to 1 & rpg4/Rpg5 & $0 ; 1$ & 0 ; to 1 & Rpg5 & 00 & 0 to $1-$ & rpg4 & $0 ; 1-$ & 0 ; to $1-$ & Rpg1 \\
\hline QSM107 & 0 & 0 & rpg4/Rpg5 & $0 ; 1$ & 0 ; to 1 & Rpg 5 & $0 ; 1$ & 0 to 2 & rpg4 & $3-3$ & $3-$ to 3 & rpgl \\
\hline QSM108 & $33-$ & $3-$ to 3 & Rpg4/rpg5 & $3-21$ & 1 to $3-$ & rpg 5 & $3-2$ & 2 to $3+$ & Rpg4 & $3-3$ & $3-$ to 3 & rpgl \\
\hline QSM109 & $3+$ & $3+$ & Rpg4/rpg5 & $3-21$ & 1 to $3-$ & $\operatorname{rpg} 5$ & $3-3$ & 2 to $3+$ & Rpg4 & $3-3$ & $3-$ to 3 & rpgl \\
\hline QSM110 & $3-$ & 2 to 3 & Rpg4/rpg5 & $10 ; 23-$ & 0 ; to $3-$ & rpg 5 & $3-2$ & 1 to 3 & Rpg4 & $3-3$ & $3-$ to 3 & rpgl \\
\hline QSM111 & $33-$ & $3-$ to 3 & Rpg4/rpg5 & $3-$ & 2 to 3 & rpg 5 & $33-$ & 2 to $3+$ & Rpg4 & $33-$ & $3-$ to 3 & rpgl \\
\hline QSM112 & $0 ; 1$ & 0 ; to 1 & rpg4/Rpg5 & 00 & 0 to 0 & Rpg5 & $0 ; 1$ & 0 to 2 & rpg4 & $0 ; 1$ & $0 ;$ to 1 & Rpg1 \\
\hline QSM113 & $0 ; 1$ & 0 ; to 2 & rpg4/Rpg5 & $0 ; 1$ & 0 ; to 2 & Rpg 5 & $0 ; 1$ & $0 ;$ to 2 & rpg4 & $0 ; 1$ & 0 ; to 1 & Rpg1 \\
\hline QSM114 & $33-$ & $3-$ to 3 & Rpg4/rpg5 & $33-$ & 2 to 3 & rpg5 & $33-$ & 2 to $3+$ & Rpg4 & $3-3$ & $3-$ to 3 & rpgl \\
\hline QSM115 & $0 ; 1$ & $0 ;$ to 2 & rpg4/Rpg5 & 00 & 0 to 1 & Rpg 5 & 00 & 0 to 2 & rpg4 & $33-$ & $3-$ to 3 & rpgl \\
\hline QSM116 & 12 & 0 ; to 2 & rpg4/Rpg5 & $0 ; 1$ & 0 to 1 & Rpg 5 & $0 ; 1$ & 0 ; to 2 & rpg4 & $3-3$ & $3-$ to 3 & rpgl \\
\hline QSM117 & $0 ; 1-$ & 0 ; to $1-$ & rpg4/Rpg5 & 00 & 0 to 0 & Rpg5 & 00 & 0 to 1 & rpg4 & $3-3$ & $3-$ to 3 & rpgl \\
\hline QSM118 & $3+$ & $3+$ & Rpg4/rpg5 & $3-3$ & $3-$ to 3 & rpg 5 & 3 & 2 to $3+$ & Rpg4 & $3-3$ & $3-$ to $3+$ & rpgl \\
\hline QSM119 & $0 ; 1$ & 0 ; to 2 & rpg4/Rpg5 & 0 & 0 & Rpg5 & $0 ; 1$ & 0 ; to $3-$ & rpg4 & $33-$ & $3-$ to 3 & rpgl \\
\hline QSM120 & 3 & $3-$ to $3+$ & Rpg4/rpg5 & $3-21$ & 1 to $3-$ & rpg 5 & $3-3$ & 2 to 3 & Rpg4 & $0 ; 1$ & 0 ; to 1 & Rpg1 \\
\hline QSM121 & $33+$ & 3 to $3+$ & Rpg4/rpg5 & $23-1$ & 1 to 3 & $\operatorname{rpg} 5$ & $33-$ & 2 to 3 & Rpg4 & $33-$ & $3-$ to 3 & rpgl \\
\hline QSM122 & $33-$ & $3-$ to 3 & Rpg4/rpg5 & $3-3$ & 2 to 3 & rpg 5 & $33+$ & $3-$ to $3+$ & Rpg4 & $3-3$ & $3-$ to 3 & rpgl \\
\hline QSM123 & 3 & 3 & Rpg4/rpg5 & $3-21$ & 1 to $3-$ & rpg 5 & $33-$ & $3-$ to $3+$ & Rpg4 & $33-$ & $3-$ to 3 & rpg1 \\
\hline QSM124 & $33+$ & 3 to $3+$ & Rpg4/rpg5 & $23-1$ & 1 to 3 & rpg 5 & $3-2$ & 2 to $3+$ & Rpg4 & $0 ; 1-$ & 0 ; to 1 & Rpg1 \\
\hline QSM125 & 0 & 0 & rpg4/Rpg5 & 00 & 0 to 1 & Rpg 5 & 00 & 0 to 2 & $r p g 4$ & $33-$ & $3-$ to 3 & rpgl \\
\hline QSM126 & $33-$ & $3-$ to 3 & Rpg4/rpg5 & $3-21$ & 1 to $3-$ & rpg5 & $3-3$ & 2 to 3 & Rpg4 & $3-3$ & 3 - to 3 & rpgl \\
\hline QSM127 & $3+$ & $3+$ & Rpg4/rpg5 & $3-3$ & 2 to 3 & $\operatorname{rpg} 5$ & $33-$ & 2 to $3+$ & Rpg4 & $0 ; 1-$ & 0 ; to $1-$ & Rpg1 \\
\hline QSM128 & 3 & $3-$ to $3+$ & Rpg4/rpg5 & $33-2$ & 2 to 3 & $\operatorname{rpg} 5$ & $33-$ & 2 to 3 & Rpg4 & $0 ; 1-$ & $0 ;$ to 1 & Rpg1 \\
\hline QSM129 & $3-3$ & 2 to 3 & Rpg4/rpg 5 & $3-21$ & 1 to $3-$ & rpg 5 & $3-3$ & 2 to 3 & Rpg4 & $0 ; 1-$ & 0 ; to 1 & Rpg1 \\
\hline QSM130 & $3-3$ & $3-$ to 3 & Rpg4/rpg5 & $213-$ & 1 to $3-$ & rpg 5 & $33-$ & 2 to $3+$ & Rpg4 & $3-3$ & $3-$ to 3 & rpgl \\
\hline QSM132 & $3-3$ & $3-$ to 3 & Rpg4/rpg5 & $3-21$ & 1 to $3-$ & $\operatorname{rpg} 5$ & $3-2$ & 1 to $3-$ & Rpg4 & $3-3$ & $3-$ to 3 & rpgl \\
\hline QSM133 & 0 & 0 & rpg4/Rpg5 & 00 & 0 to 0 & Rpg 5 & 00 & 0 to 1 & rpg4 & $0 ; 1-$ & $0 ;$ to 1 & Rpg1 \\
\hline QSM134 & $33-$ & 2 to 3 & Rpg4/rpg5 & $3-21$ & 1 to $3-$ & rpg5 & $33-$ & 2 to $3+$ & Rpg4 & $33-$ & $3-$ to $3+$ & rpgl \\
\hline QSM135 & $0 ; 1$ & 0 ; to $2-$ & rpg4/Rpg5 & 00 & 0 to $1-$ & Rpg 5 & $0 ; 1$ & $0 ;$ to 2 & rpg4 & $33-$ & $3-$ to 3 & rpgl \\
\hline QSM136 & $0 ; 1$ & $0 ;$ to 1 & rpg4/Rpg5 & $0 ; 1$ & 0 ; to 1 & Rpg5 & 00 & 0 to 1 & rpg4 & $3-3$ & 2 to 3 & rpgl \\
\hline QSM137 & $0 ; 1$ & $0 ;$ to 1 & rpg4/Rpg5 & $0 ; 1$ & 0 ; to 1 & Rpg 5 & 10 & $0 ;$ to 2 & rpg4 & $0 ; 1$ & 0 ; to 1 & Rpg1 \\
\hline QSM138 & $0 ; 1-$ & 0 ; to $1-$ & rpg4/Rpg5 & $0 ; 1$ & 0 to 1 & Rpg 5 & 00 & 0 to 0 & rpg4 & $33-$ & $3-$ to 3 & rpgl \\
\hline QSM139 & $3-3$ & 2 to 3 & Rpg4/rpg5 & $3-3$ & 2 to 3 & rpg5 & $3-2$ & 2 to 3 & Rpg4 & 12 & $0 ;$ to 2 & Rpg1 \\
\hline QSM140 & 0 & 0 & rpg4/Rpg5 & 0 & 0 & Rpg5 & $0 ; 1$ & $0 ;$ to 3 & rpg4 & $0 ; 1$ & 0 ; to 2 & Rpg1 \\
\hline QSM141 & 0 & 0 & rpg4/Rpg5 & 00 & 0 to 1 & Rpg5 & 00 & 0 to 1 & rpg4 & $3-3$ & 2 to 3 & rpgl \\
\hline QSM142 & $0 ; 1$ & 0 ; to 1 & rpg4/Rpg5 & $0 ; 1$ & 0 to 1 & Rpg 5 & $0 ; 1$ & 0 ; to 2 & rpg4 & $0 ; 1-$ & 0 ; to $1-$ & Rpg1 \\
\hline QSM143 & 3 & 3 & Rpg4/rpg5 & $213-$ & 1 to $3-$ & $\operatorname{rpg} 5$ & $33-$ & 2 to $3+$ & Rpg4 & 12 & $0 ;$ to 2 & Rpg1 \\
\hline QSM144 & $3+3$ & 2 to $3+$ & Rpg4/rpg5 & $3-21$ & 1 to $3-$ & $\operatorname{rpg} 5$ & $33-$ & 2 to $3+$ & Rpg4 & $3-3$ & $3-$ to 3 & rpgl \\
\hline QSM145 & 0 & 0 & rpg4/Rpg5 & 00 & 0 to 0 & Rpg5 & 00 & 0 to $1-$ & rpg4 & $0 ; 1$ & $0 ;$ to 1 & Rpg1 \\
\hline QSM146 & $3-$ & $3-$ & Rpg4/rpg5 & $3-$ & 2 to 3 & rpg 5 & $3-3$ & 2 to 3 & Rpg4 & $12-$ & 0 ; to 2- & Rpg1 \\
\hline QSM147 & $3-3$ & $3-$ to 3 & Rpg4/rpg5 & $3-3$ & $3-$ to 3 & rpg 5 & $3-2$ & 2 to $3-$ & Rpg4 & 10 & $0 ;$ to 2 & Rpg1 \\
\hline QSM148 & 0 & 0 & rpg4/Rpg5 & 00 & 0 to 0 & Rpg5 & 00 & 0 to 1 & rpg4 & $0 ; 1-$ & $0 ;$ to $1-$ & Rpg1 \\
\hline
\end{tabular}


indicating that a single gene in Q21861 confers resistance to race TTKSK.

The Q/SM population was previously phenotyped for resistance to $P$. graminis f. sp. tritici races QCCJ and MCCF and also $P$. graminis f. sp. secalis isolate $92-\mathrm{MN}-90(35,39)$. Raw IT data recorded on $\mathrm{Q} / \mathrm{SM}$ progeny from these evaluations are given in side-by-side comparisons with those taken for race TTKSK in Table 1 . All of the progeny exhibiting low IT modes ( 0 ; to 12$)$ to race TTKSK had similar low IT modes (00; to 10; and, rarely, 21) to $P$. graminis f. sp. tritici race QCCJ and $P$. graminis $\mathrm{f}$. sp. secalis isolate $92-\mathrm{MN}-90$. Thus, resistance to race TTKSK co-segregated with resistance to race QCCJ and isolate $92-\mathrm{MN}-90$ in the Q/SM population. Similarly, progeny exhibiting high IT modes (23- to $3+$ ) to race TTKSK also gave high IT modes to race QCCJ (3-2 to $3+3)$. A similar result was observed in response to isolate $92-$ MN-90 in that most of the 71 progeny exhibiting high IT modes to race TTKSK gave high IT modes of $23-1$ to $33-$ to the rye stem rust isolate. However, 16 of the 71 TTKSK-susceptible progeny gave lower IT modes (10;23-, 12, 21, and 213-). These progeny were still considered susceptible because the typical resistant reaction to isolate $92-\mathrm{MN}-90$ is a very low IT of 00 ; to $0 ; 1$. Thus, progeny giving an IT mode higher than $0 ; 1$ or with 3 type uredinia comprising part of its mesothetic reaction were considered susceptible. These phenotypic classifications were confirmed at the molecular level (see below).

The Q/SM population also was evaluated to race MCCF at high temperature (35). This was done to assess whether individual DH progeny also carried Rpgl because this race, when used at high temperature, is useful for detecting lines with and without the gene. The high-temperature incubation was used because resistance gene rpg4, originally found to confer resistance to race QCCJ (17), also is effective against MCCF at low incubation temperatures. Using the high incubation temperature, rpg4 is rendered completely ineffective and, therefore, lines carrying Rpg1 can be clearly resolved based on their reaction to MCCF. The presence of Rpgl had no effect on the reaction of DH progeny to race TTKSK. Similar results were previously reported in response to race QCCJ and isolate 92-MN-90 $(17,35,39)$. These data demonstrate that, although Rpgl confers resistance to many races of $P$. graminis f. sp. tritici (33), it is not effective against races TTKSK (34) or QCCJ (38). Additionally, Rpgl is generally not effective against isolates of $P$. graminis $\mathrm{f}$. sp. secalis as was previously reported $(33,38)$.

Resistance to race QCCJ in line Q21861 is conferred by a single recessive-acting gene, rpg4 (17). This gene was initially mapped to the long arm of chromosome $5 \mathrm{H}$ using random amplified polymorphic DNA (RAPD) and RFLP markers (2). In a subsequent study, Sun et al. (39) found Q21861 to be resistant to $P$. graminis f. sp. secalis isolate $92-\mathrm{MN}-90$ and demonstrated that the resistance was due to a single partially dominant gene. Resistance to isolate $92-\mathrm{MN}-90$ co-segregated with resistance to race QCCJ, prompting the authors to conclude that resistance to both formae speciales of $P$. graminis is controlled by a gene at the same locus as rpg4. In this study, resistance to race TTKSK cosegregated with resistance to both $P$. graminis f. $\mathrm{sp}$. tritici race QCCJ and also P. graminis $\mathrm{f}$. sp. secalis isolate $92-\mathrm{MN}-90$ in the $129 \mathrm{Q} / \mathrm{SM}$ progeny, indicating that the gene conferring resistance to race TTKSK also lies at the rpg4 locus. Molecular analysis of the region in the $\mathrm{Q} / \mathrm{SM}$ population positioned the locus conferring resistance to all three rusts at one crossover proximal to ABG391 and six crossovers distal to MWG740 (Fig. 1). This covers a large genomic region of approximately 5.4 centimorgans. Preliminary analysis of recombinants previously used to characterize loci conferring resistance to QCCJ and 92-MN-90 (3) suggested that the TTKSK resistance locus also resides within the narrower 70$\mathrm{kb}$ region between ARD5112 and ARD5016. This region encodes four genes in the resistant parent: Rpg5, a nucleotide-binding site leucine-rich repeat serine/threonine-protein kinase gene; rpg4, an actin depolymerizing factor-like gene; and two other genes of unidentified function (3). Positioning of the TTKSK resistance locus within the same ARD5112-ARD5016 region suggests that one or more of these genes are responsible for conferring resistance to race TTKSK. Previous high-resolution genetic and molecular analyses of the rpg4/Rpg5 region suggested that although rpg4 and Rpg5 are separate genes, both may be required for resistance to race QCCJ, whereas Rpg5 functions independently to provide resistance to isolate $92-\mathrm{MN}-90$ (3). Brueggeman et al. (4) recently suggested that resistance to race TTKSK may also involve both rpg4 and Rpg5. We can neither confirm nor refute this hypothesis based on our current data; however, this aspect is being investigated by genetic, molecular, and virus-induced gene silencing analyses.

All of the stem rust phenotyping for the Q/SM population was done on seedling plants under the controlled conditions of the greenhouse or growth chamber. A critical question to ask is whether the stem rust resistance found at the seedling stage would also be effective at the adult plant stage in the field. Adult plant resistance is essential because stem rust causes the most damage on barley after the heading stage. In field screening nurseries established at the Kenya Agricultural Research Institute in Njoro, Kenya where races TTKSK and TTKST were most common, Q21861 was highly resistant, exhibiting severities of 1 to $5 \%$ and mostly resistant to moderately resistant ITs (B. Steffenson, unpublished). The genetic basis of adult plant resistance to race TTKSK is being explored in the Q/SM population by mapping mRNA transcript abundance (i.e., expression quantitative trait loci) with the Barley1 Affymetric array (5) (R. Wise, personal communication), as was done previously for seedling resistance in the Steptoe/Morex population to race MCCF (7).

Different virulence types within the originally described race TTKS lineage (26) have been described in eastern and southern Africa $(18,19,41)$. In addition to the hallmark virulence for resistance gene $\operatorname{Sr} 31$ (16), these types also carry virulence for Sr24 (race TTKST) (18) and Sr36 (race TTKSK) (19). Wheat

TABLE 2. Primer sequences for polymerase chain reaction (PCR)-based markers in the rpg4/Rpg5 region of barley chromosome $5 \mathrm{H}$

\begin{tabular}{|c|c|c|c|c|}
\hline \multirow[b]{2}{*}{ Markers $^{\mathrm{a}}$} & \multirow[b]{2}{*}{ Primers } & \multirow[b]{2}{*}{ Sequence $5^{\prime}-3^{\prime b}$} & \multicolumn{2}{|c|}{ PCR products $(b p)^{c}$} \\
\hline & & & Q21861 & SM89010 \\
\hline \multirow[t]{2}{*}{ RB SSR1 } & RB_SSR1-F1 & CACATCCACCCATGGTTGTTGAGAG & 304 & 304 \\
\hline & RB_SSR1-R1 & CTTCACTGGTACCAGTTCGACCGAG & $\ldots$ & $\ldots$ \\
\hline \multirow[t]{2}{*}{ R4-22 STS } & $\mathrm{r} 4-\overline{\mathrm{F}} 22$ & CGGATATAGGGAGAAGGGTTTGATG & 922 & None \\
\hline & r4-R22 & GTCGTCCATCTTGTACACCACGAAC & $\ldots$ & $\ldots$ \\
\hline \multirow[t]{2}{*}{ Rpg5 STS } & QK-F6 & GGTGGATCGAAGAGAATGGAACTGC & 677 & None \\
\hline & R5-R7.3 & GCAACCTTCATTCTGACAGACCATG & $\ldots$ & $\ldots$ \\
\hline \multirow[t]{2}{*}{ R13 STS } & A12-F13 & CGCCCGACGAAAGAGAACGACAATG & 1,337 & None \\
\hline & A12-R13 & GGGCCACCGACACTGTAGCACTC & $\ldots$ & $\ldots$ \\
\hline
\end{tabular}

a Markers are designated as simple sequence repeat (SSR) or sequence tag site (STS).

b Primers are listed with 5'-3' sequences.

c Amplicon sizes are shown for each marker. All the STS markers are dominant PCR markers only amplifying a product from the resistant parent Q21861. 


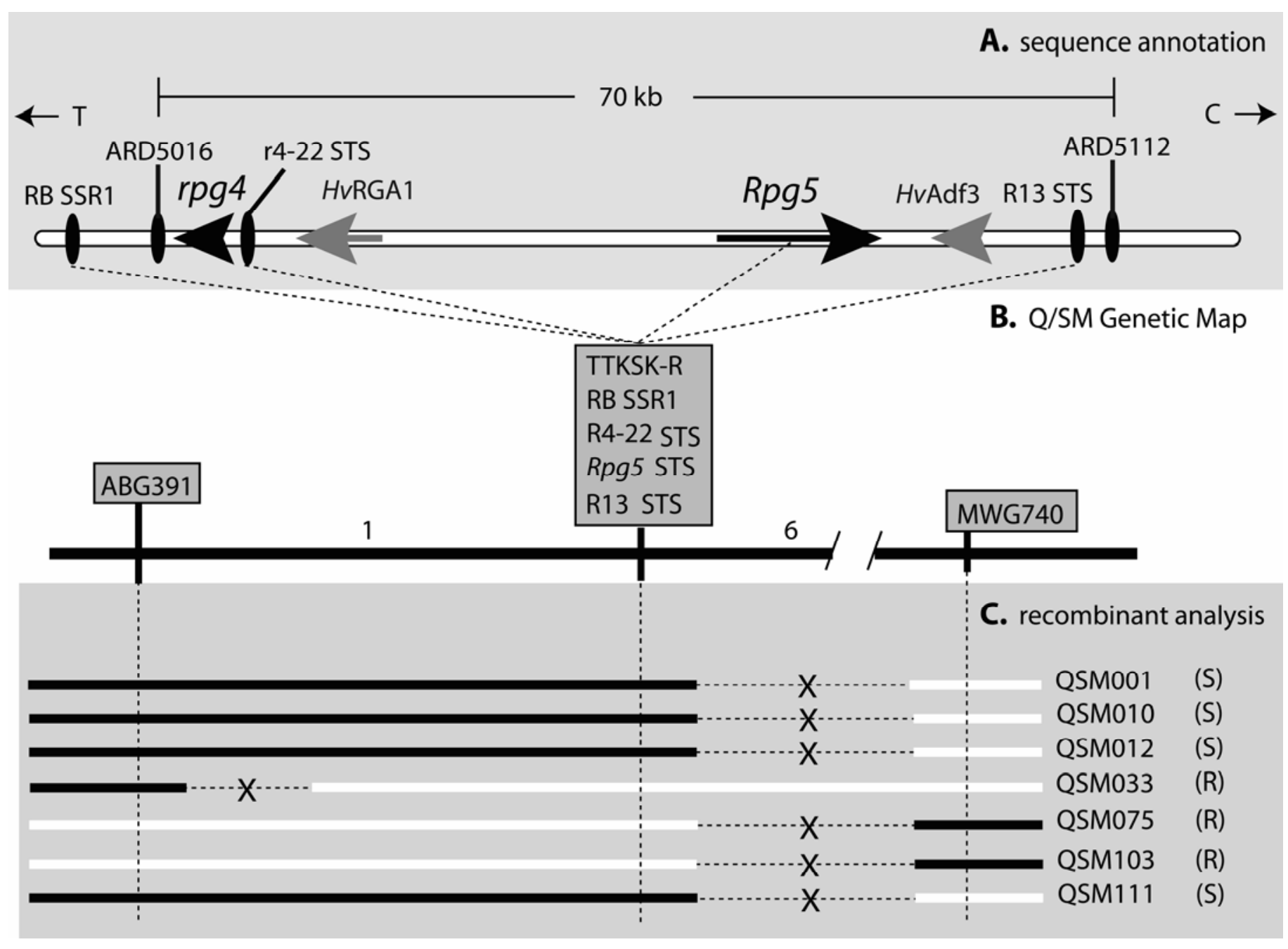

Fig. 1. Genetic and molecular analysis of the TTKSK resistance locus on barley chromosome $5 \mathrm{H}$. A, Sequence annotation of the rpg4/Rpg 5 locus from the resistant line Q21861. The white horizontal bar represents genomic sequence from line Q21861. Arrows represent annotated genes, rpg4, Rpg5 (black), and other genes (gray). Black ovals represent the position of genetic markers. ARD5016 and ARD5112 are restriction fragment-length polymorphism markers reported in Brueggeman et al. (3) delimiting the rpg4/Rpg5 region. Dashed lines connect markers and genes from sequence annotation to positions on the genetic map and recombinant analysis below. All genes and markers are labeled above. The scale is shown above in kilobases. $\mathrm{C}$ and $\mathrm{T}$ represent direction of the centromere and telomere, respectively. B, Genetic map of the rpg4/Rpg5 region from the Q21861/SM89010 (Q/SM) population. Black vertical bars represent the position of genetic and phenotypic markers present within the attached gray boxes above. The number of recombinants between markers is shown above. C, Recombinant genotype analysis. Horizontal bars represent the genotype from seven lines with recombination defining the TTKSK resistance region from the Q/SM population. White horizontal bars represent the resistant Q21861 parent and black the susceptible SM89010 parent. The dashed horizontal lines with Xs denote regions where recombination occurred. Dashed vertical lines represent the positions of connected genetic markers from the genetic map above. The recombinant designations are labeled to the right with resistant $(\mathrm{R})$ and susceptible (S), indicating response to race TTKSK.

lines carrying $\operatorname{Sr} 24$ were initially considered to be one of the best sources of resistance against race TTKSK (18). However, the identification of races with virulence for $\mathrm{Sr}_{2} 4$ in eastern Africa revealed the great vulnerability of many wheat cultivars worldwide, which are based on this resistance gene. Q21861 appears to possess a high level of adult plant resistance against both TTKSK and TTKST, two of the most common virulence types found in eastern Africa. Still, it is important to be vigilant and continually test this line against other virulence types emerging in the lineage. For the present, the stem rust resistance in Q21861 may be useful in barley breeding programs because of its effectiveness against the common African virulence types and simple inheritance. However, additional genes for resistance to race TTKSK must be identified to increase the diversity and longevity of resistance in deployed barley cultivars.

\section{ACKNOWLEDGMENTS}

This research was funded in part by the Lieberman-Okinow Endowment at the University of Minnesota. We thank S. Dahl, T. Szinyei, S.
Gale, and L. Wanshura for excellent technical assistance; B. G. Rossnagel and B. Harvey for the gift of the Q/SM population; and P. Njau, R. Wanyera, D. Singh, G. Macharia, and J. Marcharia for establishing the rust screening nurseries in Kenya.

\section{LITERATURE CITED}

1. Borlaug Global Rust Initiative. Last verified March 2009. http:// www.globalrust.org/.

2. Borovkova, I. G., Steffenson, B. J., Jin, Y., Rasmussen, J. B., Kilian, A., Kleinhofs, A., Rossnagel, B. G., and Kao, K. N. 1995. Identification of molecular markers linked to the stem rust resistance gene rpg4 in barley. Phytopathology 85:181-185.

3. Brueggeman, R., Druka, A., Nirmala, J., Cavileer, T., Drader, T., Rostoks, N., Mirlohi, A., Bennypaul, H., Gill, U., Kudrna, D., Whitelaw, C., Kilian, A., Han, F., Sun, Y., Gill, K., Steffenson, B., and Kleinhofs, A. 2008. The stem rust resistance gene Rpg5 encodes a protein with nucleotidebinding-site, leucine-rich, and protein kinase domains. Proc. Natl. Acad. Sci. USA 105:14970-14975

4. Brueggeman, R., Steffenson, B. J., and Kleinhofs, A. 2009. The rpg4/Rpg5 stem rust resistance locus in barley: Resistance genes and cytoskeleton dynamics. Cell Cycle 8:1-5. 
5. Close, T. J., Wanamaker, S. I., Caldo, R. A., Turner, S. M., Ashlock, D. A., Dickerson, J. A., Wing, R. A., Muehlbauer, G. J., Kleinhofs, A., and Wise, R. P. 2004. A new resource for cereal genomics: 22K barley GeneChip comes of age. Plant Physiol. 4:960-968.

6. Druka, A., Kudrna, D., Han, F., Kilian, A., Steffenson, B., Frisch, D., Tomkins, J., Wing, R., and Kleinhofs, A. 2000. Physical mapping of the barley stem rust resistance gene rpg4. Mol. Gen Genet. 264:283-290.

7. Druka, A., Potokina, E., Luo, Z., Bonar, N., Druka, I., Zhang, L., Marshall, D. F., Steffenson, B. J., Close, T. J., Wise, R. P., Kleinhofs, A., Williams, R. W., Kearsey, M. J., and Waugh, R. 2008. Exploiting regulatory variation to identify genes underlying quantitative resistance to the wheat stem rust pathogen Puccinia graminis f. sp. tritici in barley. Theor. Appl. Genet. 117:261-272.

8. Expert Panel on the Stem Rust Outbreak in Eastern Africa. 2005. Sounding the Alarm on Global Stem Rust: An Assessment of Race Ug99 in Kenya and Ethiopia and the Potential for Impact in Neighboring Regions and Beyond. Centro Internacional de Mejoramiento de Maíz y Trigo (CIMMYT), Mexico, DF.

9. Fetch, T. G., Jr., Johnston, P. A., and Pickering, R. A. 2009. Chromosomal location and inheritance of stem rust resistance transferred from Hordeum bulbosum into cultivated barley (H. vulgare). Phytopathology 99:339-343.

10. Fox, S. L., and Harder, D. E. 1995. Resistance to stem rust in barley and inheritance of resistance to race QCC. Can. J. Plant Sci. 75:781-788.

11. Friesen, T. L., Faris, J. D., Lai, Z., and Steffenson, B. J. 2006. Identification and chromosomal location of major genes for resistance to Pyrenophora teres in a doubled-haploid barley population. Genome 9:855-859.

12. Grando, S., and Gomez Macpherson, H. 2005. Food Barley: Importance, Uses, and Local Knowledge. International Center for Agricultural Research in the Dry Areas, Aleppo, Syria.

13. Holcomb, R., and Petrone, R. M. 2008. Agricultural Statistics 2008. National Agricultural Statistics Service (NASS). Published online by the U. S. Government Printing Office http://www.nass.usda.gov/Publications/ Ag_Statistics/2008/Chap01.pdf.

14. Jedel, P. E. 1990. A gene for resistance to Puccinia graminis f. sp. tritici in PI 382313. Barley Genet. Newsl. 20:43-44.

15. Jedel, P. E., Metcalfe, D. R., and Martens, J. W. 1989. Assessment of barley accessions PI 382313, PI 382474, PI 382915, and PI 382976 for stem rust resistance. Crop Sci. 29:1473-1477.

16. Jin, Y., Singh, R. P., Ward, R. W., Wanyera, R., Kinyua, M., Njau, P., Fetch, T., Pretorius, Z. A., and Yahyaoui, A. 2007. Characterization of seedling infection types and adult plant infection responses of monogenic $\mathrm{Sr}$ gene lines to race TTKS of Puccinia graminis f. sp. tritici. Plant Dis. 91:1096-1099.

17. Jin, Y., Steffenson, B. J., and Miller, J. D. 1994. Inheritance of resistance to pathotypes QCC and MCC of Puccinia graminis f. sp. tritici in barley line Q21861 and temperature effects on the expression of resistance. Phytopathology 84:452-455.

18. Jin, Y., Szabo, L. J., Pretorius, Z. A., Singh, R. P., Ward, R., and Fetch, T., Jr. 2008. Detection of virulence to resistance gene $S r 24$ within race TTKS of Puccinia graminis f. sp. tritici. Plant Dis. 92:923-926.

19. Jin, Y., Szabo, L., Rouse, M., Fetch, T. J., Pretorius, Z. A., Wanyera, R., and Njau, P. 2009. Detection of virulence to resistance gene Sr36 within race TTKS lineage of Puccinia graminis f. sp. tritici. Plant Dis. 93:367370.

20. Luig, N. H. 1957. Inheritance Studies of Barley in Reaction to Disease Resistance. M.S. thesis. University of Sydney, Australia.

21. Martens, J. W., Dunsmore, K. M., and Harder, D. E. 1989. Incidence and virulence of Puccinia graminis in Canada on wheat and barley in 1988. Can. J. Plant Pathol. 11:424-430.
22. Miller, J. D., and Lambert, J. W. 1955. Variability and inheritance of reaction of barley to race $15 \mathrm{~B}$ of stem rust. Agron. J. 47:373-377.

23. Nazari, K., Mafi, M., Yahyaoui, A., Singh, R. P., and Park, R. F. 2009. Detection of wheat stem rust (Puccinia graminis f. sp. tritici) race TTKSK (Ug99) in Iran. Plant Dis. 93:317.

24. Patterson, F. L., Shands, R. G., and Dickson, J. G. 1957. Temperature and seasonal effects on seedling reactions of barley varieties to three races of Puccinia graminis f. sp. tritici. Phytopathology 47:395-402.

25. Powers, L., and Hines, L. 1933. Inheritance of reaction to stem rust and barbing of awns in barley crosses. J. Agric. Res. 6:1121-1129.

26. Pretorius, Z. A., Singh, R. P., Wagoire, W. W., and Payne, T. S. 2000. Detection of virulence to wheat stem rust resistance gene $\mathrm{Sr} 31$ in Puccinia graminis f. sp. tritici in Uganda. Plant Dis. 84:203.

27. Roelfs, A. P. 1978. Estimated Losses Caused by Rust in Small Grain Cereals in the United States 1918-76. U.S. Dep. Agric. Misc. Publ. No. 1363.

28. Schafer, J. F., and Roelfs, A. P. 1985. Estimated relation between numbers of urediniospores of Puccinia graminis f. sp. tritici and rates of occurrence of virulence. Phytopathology 75:749-750.

29. Shands, R. G. 1939. Chevron, a barley variety resistant to stem rust and other diseases. Phytopathology 29:209-211.

30. Singh, R. P., Hodson, D. P., Huerta-Espino, J., Jin, Y., Njau, P., Wanyera, R., Herrata-Foessel, S. A., and Ward, R. W. 2008. Will stem rust destroy the world's wheat crop? Adv. Agron. 98:271-309.

31. Singh, R. P., Hodson, D. P., Jin, Y., Huerta-Espino, J., Kinyua, M. G., Wanyera, R., Njau, P., and Ward, R. W. 2006. Current status, likely migration and strategies to mitigate the threat to wheat production from race Ug99 (TTKS) of stem rust pathogen. CAB Rev. 1:1-13.

32. Stakman, E. C., Stewart, D. M., and Loegering, W. Q. 1962. Identification of Physiological Races of Puccinia graminis f. sp. tritici. U.S. Dep. Agric. Agric. Res. Serv. Publ. no. E617.

33. Steffenson, B. J. 1992. Analysis of durable resistance to stem rust in barley. Euphytica 63:153-167.

34. Steffenson, B. J., and Jin, Y. 2006. Resistance to race TTKS of Puccinia graminis f. sp. tritici in barley. (Abstr.) Phytopathology 96:S110.

35. Steffenson, B. J., Jin, Y., Rossnagel, B. G., Rasmussen, J., and Kao, K. N. 1995. Genetics of multiple disease resistance in a doubled haploid population of barley. Plant Breed. 114:50-54.

36. Steffenson, B. J., Olivera, P., Roy, J. K., Jin, Y., Smith, K. P., and Muehlbauer, G. J. 2007. A walk on the wild side: Mining wild wheat and barley collections for rust resistance genes. Aust. J. Agric. Res. 58:532-544.

37. Steffenson, B. J., Wilcoxson, R. D., and Roelfs, A. P. 1984. Inheritance of resistance to Puccinia graminis f. sp. secalis in barley. Plant Dis. 68:762763.

38. Sun, Y., and Steffenson, B. J. 2005. Reaction of barley seedlings with different stem rust resistance genes to Puccinia graminis $\mathrm{f}$. sp. tritici and Puccinia graminis f. sp. secalis. Can. J. Plant Pathol. 27:80-89.

39. Sun, Y., Steffenson, B. J., and Jin, Y. 1996. Genetics of resistance to Puccinia graminis f. sp. secalis in barley line Q21861. Phytopathology $86: 1299-1302$

40. U.S. Department of Agricultural, Agricultural Research Service, Cereal Disease Laboratory. Small Grain Losses Due to Rust. Last verified: March 2009. http://www.ars.usda.gov/main/site_main.htm?modecode $=36-40-05-00$

41. Visser, B., Herselman, L., and Pretorius, Z. 2009. Genetic comparison of Ug99 with selected South African races of Puccinia graminis f. sp. tritici. Mol. Plant Pathol. 10:213-222.

42. Wanyera, R., Kinyua, M. G., Jin, Y., and Singh, R. P. 2006. The spread of stem rust caused by Puccinia graminis f. sp. tritici, with virulence on Sr31 in wheat in Eastern Africa. Plant Dis. 90:113. 